\title{
Experimental Characterization of Components for Active Soft Orthotics
}

\author{
Michael Wehner \\ Yong-Lae Park \\ Conor Walsh \\ Radhika Nagpal \\ Robert J.Wood \\ Wyss Institute at Harvard University \\ Cambridge MA. USA
}

\author{
Talia Moore \\ Department of Organismic and Evolutionary Biology, \\ Harvard University, Cambridge MA. USA \\ Eugene Goldfield \\ Children's Hospital Boston \& Harvard Medical School \\ Boston, MA. USA
}

\begin{abstract}
In this paper, we present the characterization of soft pneumatic actuators currently under development for active soft orthotics. The actuators are tested statically and dynamically to characterize force and displacement properties needed for use in system design. They are shown to demonstrate remarkably repeatable performance, and very predictable behavior as actuator size (initial length) is varied to adjust total actuation. The results of the characterization are then used to inform design rules for selecting the size and number of actuators for a soft leg orthotic that can produce the force/moment/velocity properties required for a target wearer. The device was tested on a silicone model with inner frame and articulated ankle. Additionally, a portable pneumatic system was developed and characterized, that can actuate the pneumatic actuator over 800 times before refilling the gas supply.
\end{abstract}

Keywords-component; Orthotic; Soft; Pneumatics; actuator.

\section{INTRODUCTION}

Prosthetic and orthotic devices have been in use for millennia and until recently, the basic structures were for the most part quite rudimentary [1]. Over the past few decades, highly sophisticated human assistive devices, including human exoskeletons and orthotics, have begun to emerge. These have included highly engineered designs with actuation and control

This work was partially supported by the Wyss Institute for Biologically Inspired Engineering and the National Science foundation Grant (award number CNS0932015). Any opinions, findings, and conclusions or recommendations expressed in this material are those of the authors and do not necessarily reflect the views of the National Science Foundation. tuned to user requirements [2] [3] [4], and sophisticated sensing involving electromyography and suites of other sensing techniques [5] [6]. More recently, active soft orthotic devices have been developed, utilizing pneumatics and soft sensors [7].

Active soft orthotic devices offer the potential to provide a lower profile form factor than active orthotics that use more traditional robotic components, yielding a device that may be worn under the user's normal clothing. The soft nature of these devices increases user mobility and limits muscle atrophy in non-actuated degrees of freedom relative to rigid orthotics, allowing greater comfort for extended use and inclusion in a wearer's daily life. These issues of comfort, ease of use, wearer acceptance, and community acceptance become especially important for devices to be used by children. A concept sketch and photo illustrating the key components of a soft assistive orthotic are shown in Fig. 1.

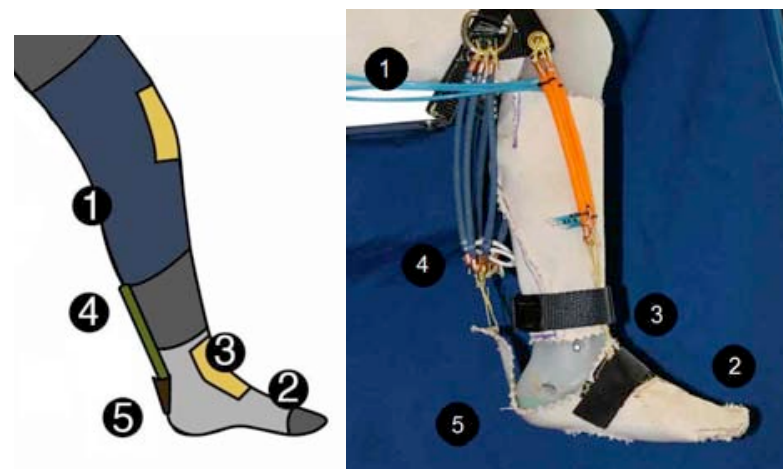

Figure 1. Concept sketch and photograph of an active soft orthotic developed using custom pneumatic actuators. 1. Body conforming soft substrate. 2. Tendon-like actuator attachment. 3. Conforming sheath based "tendon" attachment. 4. Bio-inspired actuator design. 5. Soft attachment allows passive degrees of freedom. 
In order to develop the next generation of soft orthotic devices, a thorough understanding of the constituent components must be developed. The actuators most often used in soft orthotic devices are pneumatic air-bladder actuators, commonly called McKibben actuators or Pneumatic Artificial Muscle (PAM) actuators [8]. Commercially available McKibben actuators are typically large and contain rigid components not compatible with the envisioned soft orthotic. Thus, we focus on the design and manufacture of miniature McKibben actuators that can be integrated into soft materials. With sufficient understanding of these miniaturized soft actuators, the design space for soft orthotic devices can be expanded greatly, enabling devices with improved performance, higher efficiency, and increased ergonomics leading to more natural motion for the wearer.

The primary goal of this paper is to provide a set of design tools for developing orthotics that mimic the force, velocity, moment, range, and power characteristics of natural human actions while allowing unrestricted user movement in other, non-actuated degrees of freedom. When tailored to a specific application, such as augmenting atrophied muscles, the force and actuation power of the device can be tuned to provide only the desired augmentation, reducing system size and energy requirements. We also present a portable, low profile, light-weight gas storage and distribution system to power the artificial muscles. This system is intended for a 4 year old child, with the understanding that a larger user could support the system with a larger gas canister and increased capacity. At 4 years old, a child has an established gait, and this provides a lower bound on the specifications for sizing of the components.

\section{EXPERIMENTAL CHARACTERIZATION OF Custom PNEUMATIC ACTUATORS}

We present an orthotic device developed to mimic biomechanics of dorsiflexion and plantar flexion in the gait of a healthy, $50^{\text {th }}$ percentile four year old (age and size chosen for convenience of available anatomical models and to push the form factor of possible devices smaller). Fig. 1 shows the soft orthotic device worn by a child-leg analog (elastomer structure with articulated aluminum skeletal sub-structure). This orthotic provides dorsi-flexion and plantar-flexion mimicking natural gait, and actuates inversion/eversion to the normal range of motion (quantified in section VI). Unlike most rigid orthotics, this device allows users to move freely, not being constrained by a rigid frame. This device shows one example of the type of devices possible when using these soft building blocks, with the intent that many other devices can be developed for an array of users, pathologies, and desired motions.

\section{A. Actuator Design}

McKibben-style pneumatic actuators have been designed and fabricated in various lengths using readily available commercial sub-components (Fig. 2). Functionally, the devices are composed of silicone tubing (highly extensible) housed within fiber mesh sheathing (essentially inextensible). Fittings are crimped to both ends, and holes are drilled to allow secure attachment to the orthotic. As compressed gas is valved into the system, the tubing expands, pressing against the sheathing, causing the fiber angle to change, shortening the device. Actuation travel distance can be prescribed by choosing the appropriate initial free length of the device.

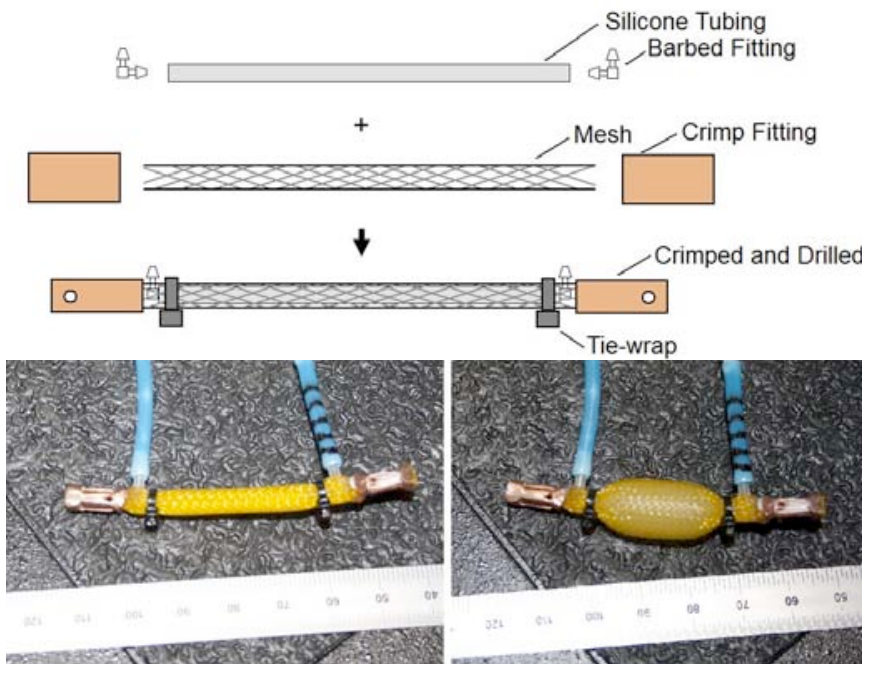

Figure 2. Pneumatic actuator. Parts and assembly diagram (top). Free state (left). Actuated (right).

\section{B. Actuator Stroke Evaluation}

Actuators of various lengths were tested to determine actuation-stroke as a function of initial free length. Fig. 3 shows the total contraction as a function of the initial actuator length. Contraction is quite consistent, near $19 \%$ for all lengths, but falls off with the shortest actuators. It is believed that end effects (ends take on hemi-spherical shape near the ends vs. a cylindrical shape through the main body) begin to play a larger role 
in shorter actuators, thus reducing overall contraction percentage.

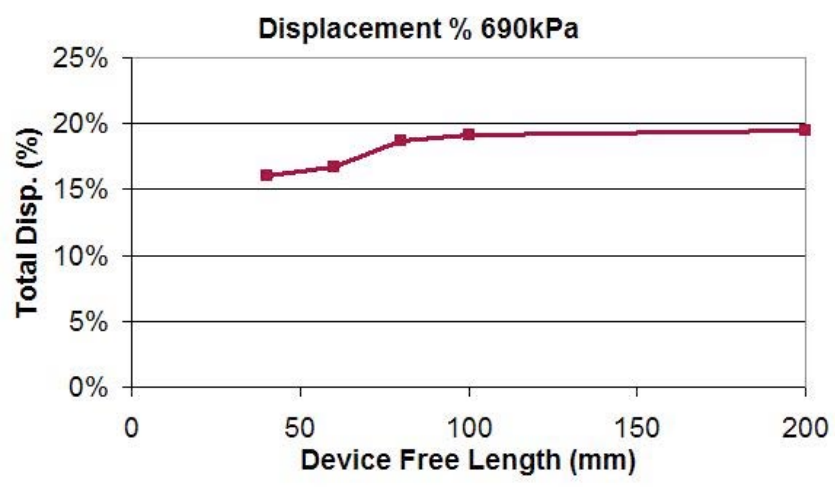

Figure 3. Actuator contraction percentage vs. device free-length.

\section{Actuator Force-Displacement Characteristics}

Force versus displacement was evaluated for various length actuators, between actuators of the same length (actuator-to-actuator) and between repeated contractions of the same actuator (run-torun). All force data was acquired at $1000 \mathrm{~Hz}$ on an Instron 5544A electromechanical testing machine.

After one "break in" contraction, which yielded lower force, the actuators exhibited very repeatable performance. A sample plot of five successive runs for a $100 \mathrm{~mm}$ actuator is shown in Fig. 4. A comparison of actuator force vs. displacement for various actuator lengths is presented in Fig. 5, showing predictable behavior based on length.

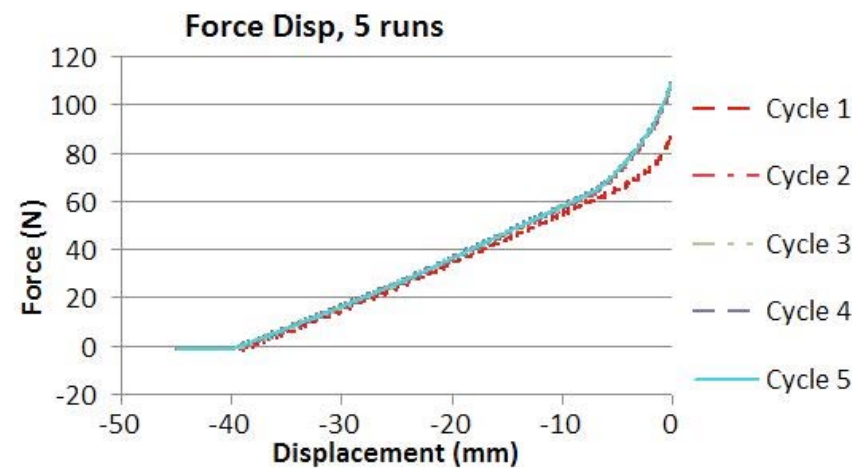

Figure 4. Repeatability of force vs. displacement of a $100 \mathrm{~mm}$ actuator for five successive runs. Neglecting the first "breaking in" contraction, One standard deviation is less than $1 \mathrm{~N}$ over $90 \%$ of curve, and less than $1.2 \mathrm{~N}$ over $100 \%$ of curve.

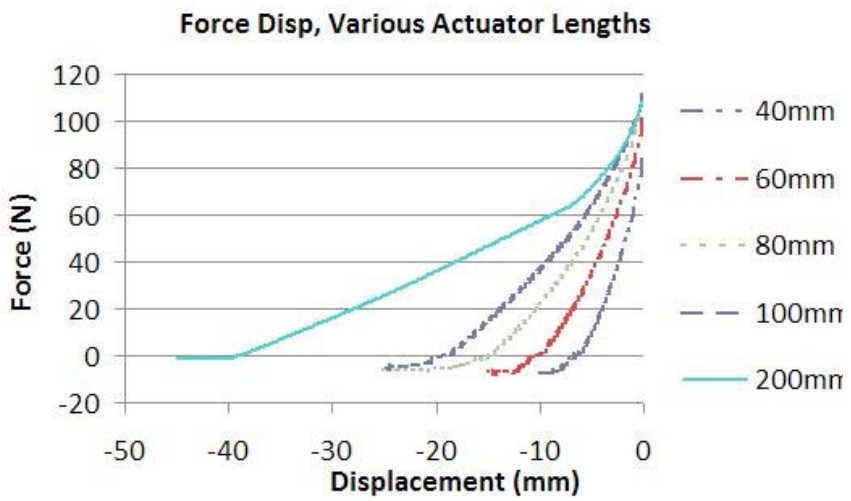

Figure 5. Force vs. displacement for various actuator lengths at $551 \mathrm{kPa}$. Force less than zero indicates contraction past the fully contracted length.

\section{Actuator Effeciency}

Actuator efficiency was calculated over the force-displacement cycle while varying velocity for actuators with lengths from $40 \mathrm{~mm}$ to $200 \mathrm{~mm}$. For these tests, the actuator was connected to the tension tester, compressed air was applied, the actuator was allowed to shorten at a prescribed rate, and the actuator was forced back to its original length at the same rate (same speed in the opposite direction). The cycle was repeated five times for each actuator, and force was recorded throughout. Again, after a first "break in" actuation, the force-displacement loops showed very repeatable behavior.

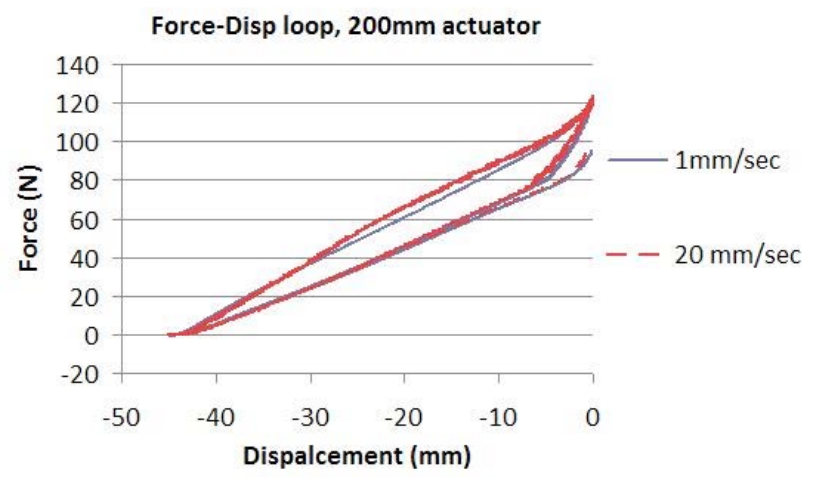

Figure 6. Force-displacement loops for a $200 \mathrm{~mm}$ actuator at two velocities ( 5 runs each). The upper curve is lengthening and the lower curve is shortening. $1 \mathrm{~mm} / \mathrm{sec}$ used as quasi-static. $20 \mathrm{~mm} / \mathrm{sec}$ represents target gait speed. All tests at $551 \mathrm{kPa}$.

Actuator loss was calculated as the area inside the loop for various length actuators at 1 and 20 $\mathrm{mm} / \mathrm{sec}$. An example plot is shown in Fig. 6. The efficiency for all actuator lengths was found to be between 65 and $75 \%$. Further, velocity was shown to have very little effect on efficiency over the range tested. 


\section{E. Actuator Dynamic Characterization}

Dynamic data was also used to calculate device power. The step response was characterized via the two methods diagramed in Fig. 7. Motion tracking was performed using video sequences acquired at 1000 frames per second and analyzed using Image software (downloaded at http://rsbweb.nih.gov/ij/). Retroflective markers (Vicon Motion Systems) were used for tracking. As shown in Fig. 8, force develops rapidly, "bounces", then increases asymptotically to a maximum value for several seconds. Waiting for this absolute maximum value to be achieved is not realistic, so times for $80 \%$ and $90 \%$ of maximum force are recorded and are shown in Table 1. We see that actuation rate increases with actuator length, particularly at higher forces.

\section{TABLE I. ACTUATION VELOCITY BY LENGTH}

\begin{tabular}{|l|l|l|l|}
\hline \multirow{2}{*}{$\begin{array}{l}\text { Time to achieve 80\% and } \\
\mathbf{9 0 \%} \text { of max force }\end{array}$} & \multicolumn{2}{|c|}{ Force (\% of max) } \\
\cline { 2 - 4 } & $\mathbf{8 0 \%}$ & $\mathbf{9 0 \%}$ \\
\hline \multirow{2}{*}{$\begin{array}{l}\text { Actuator } \\
\text { Lenth }\end{array}$} & $40 \mathrm{~mm}$ & $55 \mathrm{~ms}$ & $67 \mathrm{~ms}$ \\
\cline { 2 - 4 } & $100 \mathrm{~mm}$ & $53 \mathrm{~ms}$ & $160 \mathrm{~ms}$ \\
\cline { 2 - 4 } & $200 \mathrm{~mm}$ & $85 \mathrm{~ms}$ & $395 \mathrm{~ms}$ \\
\hline \multicolumn{2}{|l|}{} \\
\hline \multicolumn{2}{|l|}{ Actuation speed in ms. }
\end{tabular}

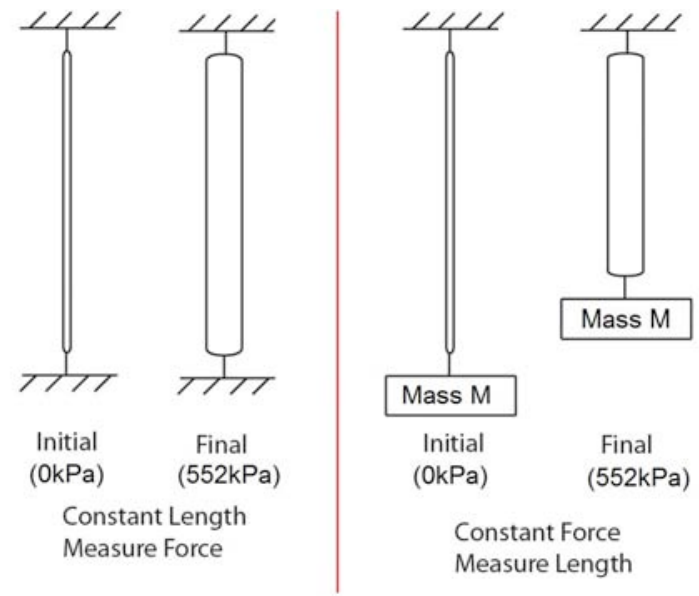

Figure 7. Step input test configurations. Left, constant length. Right, constant applied mass.

To determine the power characteristics (power output vs. applied load), masses from 1 to $5 \mathrm{~kg}$ (force 9.81 to $49.05 \mathrm{~N}$ ) were applied to the actuator, the devices were actuated, and the displacement was measured vs. time (markertracked video sequences). With constant applied force, known displacement, and known frame rate, work was calculated as force multiplied by distance per frame. Power output is then calculated by dividing by the frame rate. Fig. 9 . shows that the power output of actuators increases with applied load.

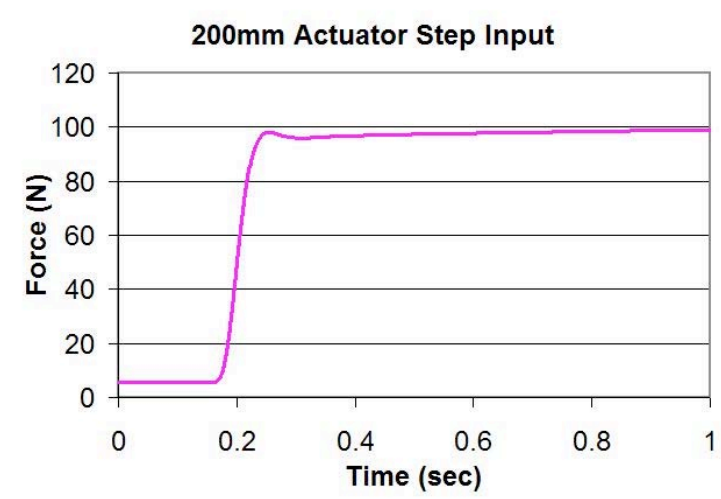

Figure 8. Sample force vs. time plot for a $200 \mathrm{~mm}$ actuator with a step input at $552 \mathrm{kPa}$. This example is from the constant length condition.

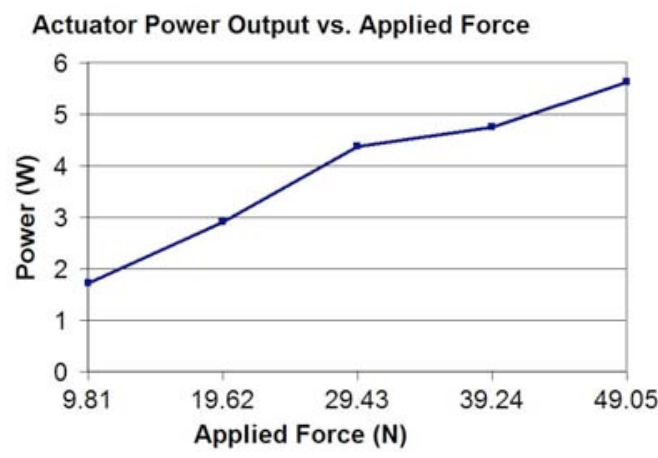

Figure 9. Power output versus applied mass. $100 \mathrm{~mm}$ actuator.

\section{Portable Power Supply}

In order to determine gas consumption of the pneumatic actuators (critical when designing a mobile gas supply), two experiments were performed. First, with devices actuated, the internal volume was calculated (through digital analysis of photographs) at a known pressure. The ideal gas law was applied to calculate the mass of the gas per actuation. Second, an air flow sensor (Honeywell AWM5104) was installed inline with exhaust plumbing and the flow was measured as actuator pressure was relieved. Integrating gas flow over eight actuation cycles yielded an average consumption of $4.64 \times 10^{-2}$ standard liters per actuation ( 0 degrees Celsius and atmospheric pressure), 1 standard deviation $8 \times 10^{-4}$ liters, or less than $2 \%$. At STP, one mole is 22.4 liters, so one cycle of a $100 \mathrm{~mm}$ actuator requires $2.07 \times 10^{-}$ 3 moles. Knowing the molar masses of air and $\mathrm{CO}_{2}$ (28.97 and 44.0096 grams respectively) we can calculate that one actuation requires $6.00 \times 10$ -2 grams of air or $9.11 \times 10^{-2}$ grams $\mathrm{CO}_{2}$. (Calculations from geometry and ideal gas law return $6.03 \times 10^{-2}$ grams of air and $9.17 \times 10^{-2}$ grams of $\mathrm{CO}_{2}$.) 
A portable gas source was developed from commercially available components (Fig. 10). The system has a mass of $1.06 \mathrm{~kg}$ and includes high-pressure and low-pressure gauges, a highpressure adjustable regulator, manual microvalves, and a fail-safe auto-off safety switch at the canister. The canister (McMaster Carr Inc.) has a mass of 304 grams, and contains 74 grams of liquid $\mathrm{CO}_{2}$ at approximately 60bar. Several components, useful during development, could be replaced with smaller devices or eliminated for a final device. Caution must be exercised in utilizing such a design, as a rapid phase change can cause the system to freeze unpredictably, possibly causing system failure. Performance characteristics will also fall off with increased rate of use, as more cold gas will be required than if the gas were near room temperature. Assuming the consumption rate is slow, and gas will be consumed isothermally, one canister will supply gas for 811 actuation cycles of a $100 \mathrm{~mm}$ actuator. More realistically, a system of actuators will work in any active device, and consumption must be calculated based on system configuration and use scenario. In the orthotic tested here, one system actuation consumed $0.278 \mathrm{~L}$ at STP $(0.55 \mathrm{~g})$, yielding a calculated value of 134 actuations per canister.

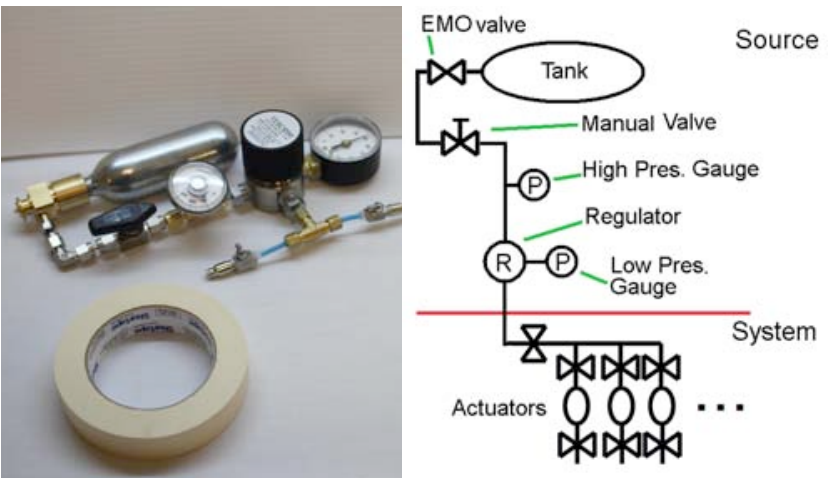

Figure 10. (left) Portable pneumatic power supply. Standard role of masking tape for size reference. (right) Pneumatic system schematic.

\section{BIOLOGICAL REQUIREMENTS FOR AN Active Soft ANKLE-FoOT ORTHOTIC}

Prior to designing an orthotic device, system requirements must be determined. For this example, we chose a leg orthotic for the ankle of a $50^{\text {th }}$ percentile 4 year-old child. The system should provide:

- Active plantar-flexion with force, power, and range equivalent to the actual child gait.
- Active dorsi-flexion with force, power, and range equivalent to the actual child gait.

- Active inversion and eversion.

- Device should not restrict natural movement in other (passive) degrees of freedom.

Anthropometry data for a child of the appropriate age was taken from CDC anthropometry study 2005 [9]. Gait data for walking was taken from Hallemans et al 2005 [10] and Human Walking [11]. The gait specifications were extrapolated to 4 year olds from the data for the somewhat younger children in [10]. Furthermore, the normal walking range of ankle motion was calculated with the knee position static. The results are summarized in Table 2.

TABLE II. ANTHROPOMETRY AND GAIT DATA

\begin{tabular}{|l|l|}
\hline \multicolumn{1}{|c|}{ quantity } & \multicolumn{1}{c|}{ value } \\
\hline Total height $(\mathrm{m})$ & 1.06 \\
\hline Total mass $(\mathrm{kg})$ & 18.1 \\
\hline Foot length $(\mathrm{m})$ & 0.16 \\
\hline Ground-knee distance $(\mathrm{m})$ & 0.31 \\
\hline Max Dorsi-flexion $\left(^{\circ}\right)$ & 5 \\
\hline Max Plantar-flexion $\left({ }^{\circ}\right)$ & 25 \\
\hline $\begin{array}{l}\text { Total travel angle } \\
(\text { Dorsi }+ \text { Plantar flexion})\left({ }^{\circ}\right)\end{array}$ & 30 \\
\hline Max moment Plantar-flexion $(\mathrm{Nm})$ & 19.95 \\
\hline Max power, Plantar-flexion $(\mathrm{W})$ & 31.7 \\
\hline Max power, Dorsi-flexion $(\mathrm{W})$ & 4.5 \\
\hline
\end{tabular}

V. Application to a Soft Ankle Orthotic

With characteristics of the actuators quantified, and anthropometry and biomechanical requirements understood, we designed a soft orthotic device to generate force, moment, and power characteristics of a $50^{\text {th }}$ percentile 4 yearold gait. Fig. 11A shows a sample foot for moment analysis. Artificial actuators exert force $F_{1}$, a distance $L_{1}$ from the center of rotation of the ankle. Distance $\mathrm{L}_{2}$ is the lateral distance from axis of rotation to the "ball" of the foot, where force is applied prior to toe-off $\left(\mathrm{L}_{2}=105 \mathrm{~mm}\right)$. Choosing a convenient distance $\mathrm{L}_{1}=45 \mathrm{~mm}$ (large enough to avoid the foot, but small enough to not require a large external lever arm), we design a stroke distance, $\Delta$, which will provide sufficient angular rotation $\left(\theta=30^{\circ}\right)$ as shown in Fig. 11B. To achieve $\theta=30^{\circ}, \Delta=23 \mathrm{~mm}$. To achieve this travel, an actuator length of $120 \mathrm{~mm}$ is required. 

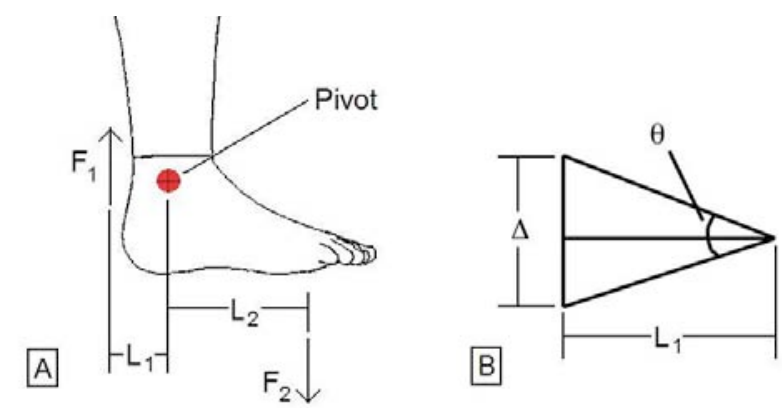

Figure 11. A. Ankle moment diagram. B. Analysis of required travel to achieve desired rotation.

Each actuator providing $100 \mathrm{~N}$ will generate a moment of $4.5 \mathrm{Nm}$. The system requires $19.95 \mathrm{Nm}$, thus 4.5 actuators are required. In order to retain symmetry, and to avoid relying on peak actuator force (force drops off quickly near free length as seen in Fig. 5), the system is designed to include 6 actuators for plantar-flexion. With regards to the power capability of the system, $120 \mathrm{~mm}$ actuators have a stroke of $24 \mathrm{~mm}$, a maximum force of $100 \mathrm{~N}$, and provide $80 \%$ of the maximum force in less than 0.1s. The peak power is thus $\mathrm{P}=24 \mathrm{~mm} \times 80 \mathrm{~N} / 0.1 \mathrm{~s}=19.2 \mathrm{~W}$ for each actuator (115.2W for 6 actuators). Section II E showed average actuation power under $5 \mathrm{~kg}$ load of $5.6 \mathrm{~W}$ (33.6W for 6 actuators). The system requires $31.7 \mathrm{~W}$ peak power, so 6 actuators will provide more than sufficient power. In addition to these quantitative system requirements, the actuators should attach in locations similar to the gastrocnemius muscles of the actual leg, while the three dorsi-flexors should attach in locations similar to the tibialis anterior, extensor digitorum longus, and superior extensor retinaculum muscles.

\section{Soft ANKLE Orthotic EXPerimental CHARACTERISTICS}

The device was attached to a custom fabricated anthropomorphic model leg that had a range of motion comparable to that during typical walking as detailed in Table 3 (note, walking range is not the maximum human body range of motion, but the maximum typical range during walking [10] [11]). The anthropomorphic model leg consists of an aluminum inner structure terminating at the ball of the foot, and surrounded by a human-like silicone form, providing a rigid inner structure from the heel to the ball of foot which had a passive degree of freedom. All aluminum components are wrapped in cotton cloth fixed to the structure with screws to minimize delamination between metal and silicone. Dorsiflexion actuators were attached to Kevlar line acting as tendon analogs, and routed through short Teflon tubes, attached in locations similar to human tendon sheathing. A spread-harness configuration at the muscle origin points near the knee was used to spread forces, minimizing local contact pressure. Finally, the Kevlar line was attached to a distributed network of Kevlar fibers spreading actuator force across the area of tendon insertion (Fig. 12). The ankle and foot joints were manually moved through their degrees of freedom with minimal impedance from the orthotic device.

TABLE III. WALKING RANGE OF MOTION VS. DEVICE RANGE.

\begin{tabular}{|c|c|c|}
\hline & during walking & leg model \\
\hline dorsiflexion $\left({ }^{\circ}\right)$ & 5 & $>30$ \\
\hline plantarflexion $\left({ }^{\circ}\right)$ & 25 & $>30$ \\
\hline inversion $\left({ }^{\circ}\right)$ & 5 & $>10$ \\
\hline eversion $\left({ }^{\circ}\right)$ & 5 & $>10$ \\
\hline \multicolumn{3}{|c|}{$\begin{array}{l}\text { *total range of motion is the sum of opposing ranges. Total } \\
\text { dorsiflexion-plantarflexion is }>60^{\circ} \text {, total inversion-eversion range } \\
\text { is }>20^{\circ} \text {. }\end{array}$} \\
\hline
\end{tabular}

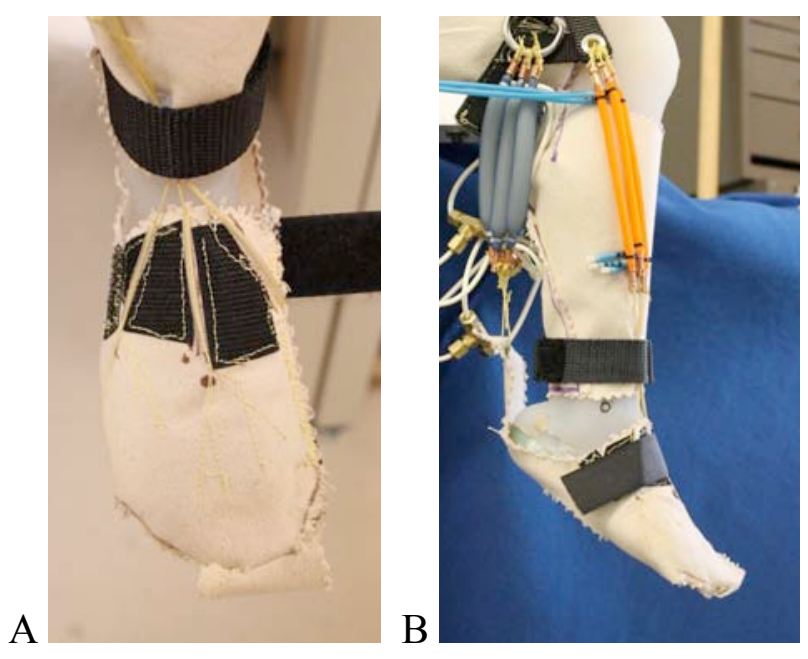

Figure 12. Actuator insertion locations. A. dorsi-flexors. B. PlantarFlexors.

A custom fixture was designed to attach to the leg with a load cell so that force data could be recorded on the forefoot as plantar-flexors were actuated. Local deformation of the elastomeric leg model above the knee caused the orthotic device to flex near the muscle origin points, reducing maximum moment to $14.2 \mathrm{Nm}$ for an input pressure of $552 \mathrm{kPa}$. Pneumatic pressure was increased to $689 \mathrm{kPa}$, and achieved $21.5 \mathrm{Nm}$, shown in Fig. 13, greater than the goal of $19.95 \mathrm{Nm} .80 \%$ of the maximum moment was achieved in $98 \mathrm{~ms}$, and $90 \%$ in $117 \mathrm{~ms}$. 
The total ankle range of motion was determined through digital analysis of photographs, and determined to be 33.5 degrees total travel in dorsi/plantar flexion (variable depending on tendon length). Similarly, inversion/eversion was measured as 18.8 degrees total travel. Sample images of neutral and flexed states are shown in Fig. 14.

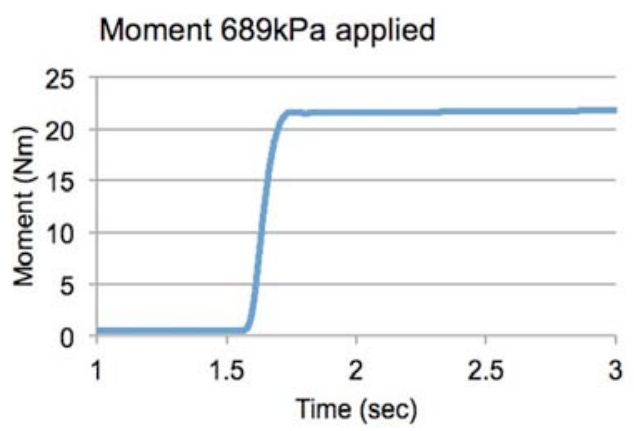

Figure 13. Ankle moment vs time for 6 actuators at $689 \mathrm{kPa}$ pneumatic pressure.

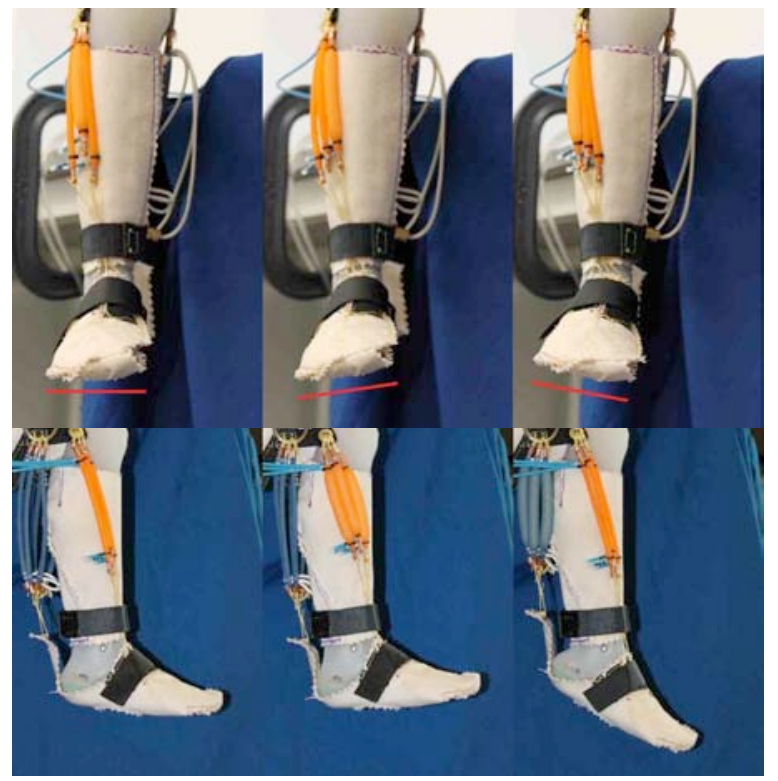

Figure 14. Orthotic device, (Top 1-r) Neutral, Inversion, Eversion. (Bot 1-r) Neutral, Dorsiflexion, Plantarflexion

\section{CONCLUSION AND FutURE WORK}

The main contributions of this work are the static and dynamic characterization of force and displacement for miniature pneumatic actuators that are suitable for active soft orthotic designs. Using the results of this work, we present a case study of an ankle-foot active soft orthotic and demonstrate that we can achieve the kinematics and kinetics required supporting walking of a 4 year old. This successful demonstration on an articulated leg model validates our use of this characterization data as part of the design process for these types of devices.

Future work will be undertaken to reduce flow (extend $\mathrm{CO}_{2}$ life) by reducing the number of actuators, develop a better leg analog to more closely emulate physical properties of human leg (less local deformation at muscle origins), and integrate sensors and regenerative pneumatics. Furthermore, to assist pathologies characterized by known joint offsets such as excessive plantar flexion in cerebral palsy patients, orthotic designs can include a constant restoring force in addition to soft actuators, allowing a full range of motion with reduced complexity and energy consumption. Though comfort, ease of use, and acceptance is believed to be improved with soft systems, human subjects research is required to evaluate these factors in a systematic and controlled manner.

\section{References:}

[1] A. Thurston, "Pare and prosthetics: the early history of artificial limbs," ANZ Journal of Surgery, Volume 77, No. 12, Blackwell Publishing Asia, 2007, pp1114-1119.

[2] C. Walsh, K, Endo, and H, Herr, "A quasi-passive leg exoskeleton for load-carrying augmentation," International Journal of Humanoid Robotics Vol. 4, No. 3, World Scientific Publishing Company, 2007, pp. 487-506.

[3] J. A.Blaya; H. Herr, "Adaptive control of a variable-impedance ankle-foot orthosis to assist drop-foot gait," Neural Systems and Rehabilitation Engineering, IEEE Transactions on , vol.12, no.1, pp.24-31, March 2004

[4] M. Wehner, D. Rempel and H. Kazerooni, "Lower Extremity Exoskeleton Reduces Back Forces in Lifting" ASME Dynamic Systems and Control Conference October 12-14, 2009 , Hollywood, California, USA pp. 49-56.

[5] M. Wehner, (2012) "Man to machine, applications in electromyography," EMG Methods for Evaluating Muscle and Nerve Function. Intech Publishing, retrieved January 22, 2012, from http://www.intechopen.com/articles/show/title/man-to-machineapplications-in-electromyography

[6] A. Chu, H. Kazerooni, and A. Zoss, "On the Biomimetic Design of the Berkeley Lower Extremity Exoskeleton (BLEEX)," Robotics and Automation, 2005. ICRA 2005. Proceedings of the 2005 IEEE International Conference on Robotics and Automation, pp. 43454352, 18-22 April 2005

[7] Y.-L. Park, B. Chen, D. Young, L. Stirling, R. Wood, "Bio-inspired Active Soft Orthotic Device for Ankle Foot Pathologies," EEE/RSJ International Conference on Intelligent Robots and Systems, San Francisco CA. pp. 4488 - 4495, Sept. 2011

[8] K. Ahn, H. Nguyen, "Intelligent switching control of a pneumatic muscle robot arm using learning vector quantization neural network," Mechatronics, Volume 17, Issues 4-5, May-June 2007, Pages 255262.

[9] M. McDowell, C. Frayar, R. Hirsch, C. Ogden "Anthropometric Reference data for Childern and Adults: U.S. Population, 19992002" Vital and Health Statistics, Center for Disease Control, July, 2005.

[10] A. Hallemans, D. De Clercq, B. Otten, P. Aerts, 3D joint dynamics of walking in toddlers A cross-sectional study spanning the first rapid development phase of walking. Gait \& Posture 2005, 22:107118.

[11] J. Rose, J. Gamble, Human Walking, 3rd ed. Philadelphia, PA. Lippincott Williams \& Wilkins, 2006, pp 64-72 